\title{
Comparativestudy Between Tramadol Hydrochloride and Sodium Hyaloronate for Management of Tempromandibular Joint Internal Derangement
}

\author{
Yasser Mohammed El-Gerby, Mohammed Ahmed El-Sholkamy, Eman Abdelhalim El-Sharrawy \\ Oral and Maxillofacial Surgery,Faculty of Dentistry,Suez Canal University, Eygpt \\ Email address: \\ yaserelgerby@yahoo.com (Y. M. El-Gerby) \\ To cite this article: \\ Yasser Mohammed El-Gerby, Mohammed Ahmed El-Sholkamy, Eman Abdelhalim El-Sharrawy. Comparative Study Between Tramadol \\ Hydrochloride and Sodium Hyaloronate for Management of Tempromandibular Joint Internal Derangement. Advances in Surgical \\ Sciences.Vol. 3, No. 3, 2015, pp. 19-26.doi: 10.11648/j.ass.20150303.11
}

\begin{abstract}
Purpose. This study was conducted to evaluate the efficacy and safety of arthrocentesis with injection of tramadol hydrochloride for management of tempromandibular joint with internal derangement compared with traditional method with sodium hyaloronate injection. Patient\& methods. The study was conducted on forty patients classified as class ASA type I with chief complain of limited mouth opening, TMJ pain, and clicking sounds in the TMJ was included in this study. All patients included in the study was selected from the outpatient clinic of oral and maxillofacial department, faculty of dentistry, Suez canal university. The selected patients were divided randomly into two equal groups, group I:consists of 20 patients where arthrocentesis was performed for the affected joint followed by in traarticular injection of one ml, commercially available tramadolhydrochloride. GroupII: consisted of 20 patients where arthrocentesis was performed for the affected joint followed by intraarticular injection of one $\mathrm{ml}$. commercially available sodium hyaloronate. Evaluation of the patients was done preoperatively, after1,3,6 months postoperatively which included maximal mouth opening, pain\& clicking sound presence in TMJ. Results. patients with either disc displacement with reduction or without reduction were benefited from the arthrocentesis procedure with injection of Sodium Hyaluronate and also with tramadol hydrochloride. Both treatments were able to reduce pain levels, increase maximal mouth opening and reduce clicking withstatistically significant difference. However, arthrocentesis with injection of tramadol hydrochloride was superiorto arthrocentesis with injection of sodium hyaluronate in terms of treatment of internal derangements of the temporomandibular joint. Conclusion. It has been concluded arthrocentesis is an effective conservative procedure in treatment of temporomandibular joint internal derangement, clinical efficacy of arthrocentesis with tramadolhydrochloride in the temporomandibular joint internal derangements\& safty of tramadolintraarticular injection in the temporomandibular joint.
\end{abstract}

Keywords: Sodium Hyaloronate, TramadolHydrochloride, Arthrocentesis, Internal Derangement

\section{Introduction}

Internal derangements of the Temporomandibular Joint (TMJ) are characterized by displacement of the intra-articular disc, which acts as an obstacle to normal joint movement, it results in clicking and popping sounds or locking and an inability to open the mouth widely. These conditions may be associated with pain, especially during function, themost common causes are trauma or chronic parafuntion. (Laskin,2009).

Nitzan et al., (1991)described a technique of irrigation of the upper compartment of the Temporomandibular Joint (TMJ) with Ringer's lactate solution to treat limited mouth opening due to internal derangement. They reported an increase in mouth opening and decrease of pain. This technique marked an evolution towards less surgical treatment.

Predictor variables for treatment outcomes of arthrocentesis and hydraulic distention of the temporomandibular joint. They investigated whether the clinical variable of TMJ pain chronicity and the magnetic resonance imaging variable of osteoarthrosis may predict treatment outcomes of arthrocentesis and hydraulic distention of the TMJ. They concluded that arthrocentesis in chronic TMJ pain patients was less successful than in no nchronic patients with regard to treatment outcome of pain reduction. Arthrocentesis in patients with TMJ osteoarthrosis was more beneficial with regard to 
the disappearance of disc displacement without reduction than in non-osteoarthrosis patients. Emshoffand Rudisch(2004).

Emshoff (2005), stated arthrocentesis, is more successful in subjects who are less than 25 years old and who exhibit VAS pain scores $>5$ with MMO less than $25 \mathrm{~mm}$. This also suggests the efficiency of arthrocentesis for the management of TMJ disorder.

Introduction of hyaluronic Acid injection after arthrocentesis to restore joint lubrication, were expected to extend to other TMJ disorders, and encouraging findings were reported in patients with inflammatory-degenerative disorders as well as internal derangements. Hyaluronic acid HA is a high molecular weight polysaccharide and a major natural component of synovial fluid. The importance of HA in the lubrication of synovial tissues has been established but its function inrelation to the occurrence of joint diseases is not precisely known. HA is largely responsible for the viscosity of normal synovial fluid. HA is a good soft tissue lubricant under loads and has been reported to prevent intra-articular adhesions. Guarda et al. (2007).

Arthrocentesis of the temporomandibular joint disorders, Kaneyamaet al(2004) In their study to investigate the ideal volume of perfusate for found that arthrocentesis is effective for washing out bradykinin, interleukin-6, and protein from the $\mathrm{TMJ}$, and the ideal lavage volume of perfusate for arthrocentesis is between 300 and $400 \mathrm{~mL}$.

The relationship between effectiveness of arthrocentesis under sufficient pressure and conditions of the temporomandibular joint. They found that the pathologic conditions of the TMJ did not have an influence on the efficacy of arthrocentesis under sufficient pressure. They suggested that arthrocentesis under sufficient hydraulic pressure has wider application than arthrocentesis under low pressure. Yuraand Totsuka(2005).

Arthrocentesis is an easy, minimally invasive, highly efficient procedure to decrease joint pain and increase the range of mouth opening in patients with closed lock of the temporomandibular joint. They described it as the simplest form of surgery in the TMJ, aiming to release the articular disc and to remove adhesions between the disc surface and the mandibular fossa by means of hydraulic pressure from irrigation of the upper chamber of the TMJ. The adhesions are released after irrigation of the upper joint space under sufficient hydraulic pressure.Alkan and Kilic( 2008).

El-Sharrawy EAet al, (2006) who stated thatantiinflammatoryeffects of tramadol and a clinical advantages regarding safety or efficacy for treating postsurgical dental pain.

Hanife A. et al (2010) conclude that the IA tramadoladministered postoperatively provided a significant analgesic benefit and decreased the opioid requirements after arthroscopic knee surgery.

Roy M. F. et al (2001) who stated that tramadol has an effective adjunct to NSAIDs for the treatment of OA pain. The results of this study support the usefulness of tramadol as monotherapy in the treatment of patients with joint pain associated with $\mathrm{OA}$.

\section{Patients and Methods}

After approval of an ethical committee \&obtaining an informed concent of all patients, the study was conducted on forty patients were divided randomly into two equal groups, The study was conducted on forty patients classified as class ASA type I with chief complain of limited mouth opening, TMJ pain, and clicking sounds in the TMJ was included in this study. All patients included in the study was selected from the outpatient clinic oforal and maxillofacial department, faculty of dentistry, Suez canal university.

Patients with history of degenerative joint disease, or those who have performed previous surgical treatment was excluded from this study.

The selected patients were subjected to the following assessment:-

Preopertive assessment preparationfor every patient by using printed questionnaire sheet.

A- Clinical evaluation:

All patients had a standard examination before surgery. They completed a visual analog scale (VAS) to assess pain.The maximum interincisal mouth opening was measured preoperatively with a caliber to the nearest millimeter.Joint sounds were evaluated preoperatively by finger palpation and a stethoscope.

B- Radiographic evaluation:

All patients were evaluated radiographically before the surgical procedures. The radiographic examination included TMJ view \&MRI.Fig. (1,2\&3)

Based on these examination and patient's history, a diagnosis of TMJ internal derangement was made.

The selected patients were divided randomly into two equal groups

Group I:This group consists of 20 patients where arthrocentesis was performed for the affected joint followed by intraarticular injection of one $\mathrm{ml}$, commercially available tramadol. Fig. 4.

Group II: This group consists of 20 patients where arthrocentesis was performed for the affected joint followed by intraarticular injection of one $\mathrm{ml}$. commercially available sodium hyaloronate.Fig. 5.

Arthrocentesis technique:-

All patients were informed about the procedure, its possible complications, and about the materials used. The operative site was prepared aseptically using Betadine solution and the area was isolated with sterile drapes. fig 6 .

The point of needle insertion was determined by drawing the canthal-tragus line and a point $10 \mathrm{~mm}$ in front of the tragus and $2 \mathrm{~mm}$ below the canthal-tragus line was marked. Another point $2 \mathrm{~mm}$ anterior to the former one was marked to serve as the point of insertion of the second needle used for collecting the synovial fluid aspirates.

For the auriculotemporal nerve block 0.3 to $0.5 \mathrm{ml}$ of an anesthetic solution was injected and then the needle was introduced into the upper joint compartment and approximately $3.5 \mathrm{ml}$ of anesthetic solution was injected. 
The TMJ was palpated and the upper joint space enlarged by downward and forward displacement of the mandible.Hydraulic pressure was created by injecting about $2 \mathrm{~mL}$ of saline solution into the space. A second 20 -gauge needle was placed approximately $2 \mathrm{~mm}$ anterior to the former needle to establish outflow. The joint was then lavaged with $300-500 \mathrm{~mL}$ of saline solution injected into the upper joint compartment. The outflow needle was periodically occluded in order to create hydraulic pressure within the joint space.Fig. 7.

On termination of the procedure, one $\mathrm{ml}$ of commercially available (Amadol ampoules I.M/I.V 100mg/2ml).produced by ADWAIA-EYGPT. was injected in the joint of 20 patients represented group I., while in group II, one $\mathrm{ml}$ of commercially available Sodium Hyaluronate (curavisc 20mg / $2 \mathrm{ml}$ syringe)by IDTBiologika GmbH campany, Germany was injected. Fig. 8\&9.

After removal of both needles the mandible was gently manipulated in order to evaluate joint movement.The patients practiced opening the mouth as wide as they could. After the arthrocentesis, They also were instructed for active and isometric opening exercises, protrusive, and lateral excursive exercises. Antibiotics were prescribed to be taken 3 times daily for 3 days. Evaluation of the patients was done preoperatively, after1,3,6 months postoperativelywhichincludedmaximal mouth opening, range of mouth opening which measured and compared preoperatively and postoperatively. Pain was recorded on $100 \mathrm{~mm}$ visual analog scales \& mandibular function and presence of TMJ sounds both preoperatively and postoperatively.

\section{Results}

\subsection{Evaluation of Pain}

Comparison between both groups:

Preoperative:-

There was no significant difference between both groups as pain score of group I was $(8.10 \pm 0.91) \&$ group II was $(8.10 \pm$ $0.79)$ where $(\mathrm{P}$ value $=1.000)$. Table 1,Figure 10.

At one month postoperative:-

There was high significant reduction in pain score levelbetweenboth groups as in group I was $(4.25 \pm 0.64) \&$ in group II was $(3.65 \pm 0.49)$ as $(\mathrm{P}$ value $=0.000)$. Table 1 , Figure 10.

At three months postoperative:-

There was high significant reduction in pain score level in both groups as in group I was $(1.65 \pm 0.49) \&$ in group II was $(5.80 \pm 0.77)$ as $(\mathrm{P}$ value $=0.000)$. Table 1,Figure 10 .

At six months postoperative:-

There was high significant reduction in pain score level in both groups as in group I was $(0.80 \pm 0.62) \&$ in group II was $(2.30 \pm 0.66)$ as $(\mathrm{P}$ value $=0.000)$. Table 1, Figure 10.

\subsection{Maximal Mouth Opening}

Comparison between both groups:

\section{Preoperative:-}

There was no significanant difference between two groups as mouth openingofgroup Iwas $(17.30 \pm 2.85) \&$ group II was $(16.70 \pm 3.29)$ where $(\mathrm{P}$ value $=0.542)$. Table 2,Figure 11 .

At one month postoperative:-

There was high significant increase in mouth openingin both groups as in group I was $(37.95 \pm 3.22) \&$ in group II was $(47.50 \pm 3.69)$ as $(\mathrm{P}$ value $=0.000)$. Table 2 , Figure 11.

At three months postoperative:-

There was high significant increase in mouth opening in group I was $(45.30 \pm 2.79) \&$ while significant decreasein group II was $(42.35 \pm 3.65)$ as $(\mathrm{P}$ value $=0.007)$. Table 2 , Figure 11.

At six months postoperative:-

There was high significant increase in mouth opening in group I was $(49.80 \pm 2.19) \&$ while significant decreasein group II was $(38.15 \pm 3.54)$ as $(\mathrm{P}$ value $=0.000)$. Table 2, Figure 11.

\subsection{Clicking Sound}

In the current study for assessment of clicking sound, the study groups were divided into patients with joints presented with clicking and others without clicking. Table 3,Figure 12.

In the group I, it was reported that 5 patients with joints presented without clicking (25\%) and 15 patients withjoints with clicking $(75 \%)$ of total 20 patients.

In the group II, it was reported that 2 patients with joints presented without clicking $(10 \%)$ and 18 patients withjoints with clicking $(90 \%)$ of total 20 patients.

After one month, in group I clicking was reported in $50 \%$ while in group II clicking where reduced in to $25 \%$. Table 4,Figure 12.

After three months, in group I clicking was reportedin $35 \%$ while in group II clicking where elevated to $35 \%$. Table 5,Figure 12.

After six months, in group I clicking was reported in $25 \%$ while in group II clicking where continuous elevation to $50 \%$. Table 6,Figure 12.

\section{Discussion}

Temporomandibular joint diseases are common disorders in the population and they may strongly affect the quality of life. The term "internal derangement of the TMJ" expresses an abnormal relationship among mandibular condyle, articular eminence, and articular disc. It is usually characterized by pain, joint noises, and sometimes by limitation of jaw movements. Arthrocentesis of the TMJ is commonly defined as a lavage of the joint and is traditionally accomplished without viewing the joint. (Gulen H. et al, 2009)

Temporomandibular joint arthrocentesis is usually an officebased procedure. Although it has been widely used for various TMJ disorders andits action mechanism is still not clear. It was proposed that lavage and lysis of the upper joint compartment would eliminate the vacuum effect, resolve the adhesions, and alter the viscosity of the synovial fluid thereby aiding 
translation of the disk and condyle.Arthrocentesis is also said to solve three common symptoms: limitation in mouth opening in closed lock cases, pain, and dysfunction. It is believed that washing out of inflammatory mediators is effective in pain reduction, as well. (Kaneyama K et al, 2004 ).

In The current study fourty patients suffered from limited mouth opening and pain in their temporomandibular joints were treated with arthrocentesis. All cases were treated under local anesthesia in the outpatient clinic. Pain level on the visual analogue scale VAS, maximal mouth opening and joint clicking were the evaluation criteria for all patients included.

Most of publications depended on the visual analogue scale, Maximal interincisal mouth opening and jaw function as evaluation criteria of arthrocentesis. Sixteen clinical articles regarding arthrocentesis were reviewed by Monje et al (2012). A series of clinical and procedure variables were analyzed. Success was considered to be "changing the impaired mandibular function in sufficient measure" as the result of restored movement and reducing pain in the TMJ. The overall success rate was $83.5 \%$. Consequently, they concluded that arthrocentesis is a simple, non-invasive, inexpensive and highly effective procedure, apart from having a low morbidity rate also they suggested that arthrocentesis should be considered as an alternative to other more invasive TMJ surgical procedures, provided it is applied to selected groups of patients.

In the present study arthrocentesis was performed for all patients. In group I, patients were subjected to arthrocentesis with tramadol injection, In groupII, patients were subjected to arthrocentesis with Sodium Hyaluronate injection. The patients were followed up for six months and the results revealed the efficacy of arthrocentesis as there were a significant improvement in Pain, maximum mouth opening\&clicking sound. Accordingly our results in group I \& group II indicate the efficacy of arthrocentesis as a simple noninvasive effective procedure in the treatment of the temporomandibular joint internal derangement. These results were in agreement with Neeli et al. (2010) as they evaluated the efficacy of arthrocentesis in the treatment of internal derangement of the temporomandibular joint (TMJ).In their study thirty patients with TMJ internal derangement underwent arthrocentesis using saline. Pain using visual analog scale, maximum mouth opening, joint noises and mandible deviation were documented pre-operatively, postoperatively and monthly followed up till one year. $96 \%$ of cases showed a significant reduction in pain with arthrocentesis. Pain reduction is attributed to the high pressure irrigation which washes away inflammatory mediators and providing pain relief. They suggested also arthrocentesis under sufficient pressure can remove adhesions, widen the joint spaces and improve mouth opening. In patients who presented with limited mouth opening, significant improvement was seen in the immediate post-operative period and with reduction in pain, mouth opening further increased from third to sixth month. Improvement was observed with jaw deviation and clicking.

Hyaluronic acid is a natural component of joint synovial fluid and is also found in the extracellular matrix of connective tissue. The excellent mechanical and metabolic properties of its molecules define it as an ideal treatment for inflammatory problems of the temporomandibular joint. Hyaluronic acid has been proposed as an alternative therapeutic agent with same therapeutic effect, that improve and restore normal lubrication in joints, provides nutrition to the vascular articulating disc and stabilize the joint.It has been widely used in the intraarticular injection for patients with temporomandibular joint dysfunction as it improves function and reduce pain. Escoda et al (2010)

In the current study, all patients in group II were subjected to arthrocentesis followed by injection of sodium hyalurinate. Patients were followed up for six months. There was a significant reduction in pain with increase in the range of maximal mouth opening. These results were in accordance with Manfredini et al. (2012) who stated that, the first attempts on TMJ arthrocentesis focused on its application to increase jaw function and achieve relief from pain in patients with restricted mouth opening. Then, with the increase in knowledge on the role of joint lubrication impairment as a risk factor for TMJ internal derangements, viscosupplementation with hyaluronic acid became an option for the management of symptoms in the clinical setting. This led to the progressive expansion of potential clinical indications for the use of arthrocentesis plus hyaluronic acid injection, with particular regard to joints with inflammatory-degenerative disorders \&in accordance to the study which was introduced by Bjørnland et al (2007) as they compared the efficacy and complications of intra-articular TMJ injections with either sodium hyaluronate or corticosteroids. They reported that, injections with a high molecular weight hyaluronic acid were significantly more effective in decreasing pain intensity than injections of corticosteroids in osteoarthritic joints.

In the current study, all patients in group I were subjected to arthrocentesis followed by injection of tramadol injection. Patients were followed up for six months. There was a significant reduction in pain with increase in the range of maximal mouth opening. These results were in accordance with van Loon J P et al (2012) who stated that intra-articular (IA) administration of opioids has been shown to provide analgesic and antiinflammatory effects during acute and chronic pain in human joints $\&$ also in accordance with Tamer A.H (2012) who concluded that intraarticular injection of tramadol is effective in management of clinical symptoms associated with internal derangements of the tempromandibular joint, Although tramadol showed a superior results over COX-2 inhibitors yet, arthrocentesis with intrarticular injection of COX-2 inhibitors represents a possible choice for patients with internal derangements of the tempromandibular joint \& also in accordance with Hanife A. et al (2010) conclude that the IA tramadoladministered postoperatively provided a significant analgesic benefit and decreased the opioid requirements after arthroscopic knee surgery\& in agreement withKapralS. et al (1999) who concluded that there is a limited absorption of the drug and that the mechanism of the analgesic effect of intraarticularly 
administered tramadol is not due to the systemic effects \&in accordance withRoy M. F. et al (2001) who stated that tramadol has an effective adjunct to NSAIDs for the treatment of OA pain. The results of this study support the usefulness of tramadol as monotherapy in the treatment of patients with joint pain associated with $\mathrm{OA}$.

In the current study, values of sodium hyalurinate group in maximal mouth opening were higher than tramadol group with high statistical significance after one month postoperatively but the values of sodium hyalurinate group in maximal mouth opening were lower than tramadol group with high statistical significance after three \&six months postopertively. This indicated the efficacy of tramadol in improving jaw functions while the superiority of sodium hyalurinate injection with arthrocentesis in improving jaw functions may be attributed to the topical and lubricant action of sodium hyalurinate.

These results were in accordance with the results obtained by Yeunget al (2006), they studied Short-term therapeutic outcome of intra-articular high molecular weight hyaluronic acid injection for non-reducing disc displacement of the temporomandibular joint. In their study,mouth opening was noted to decrease when compared to pre-injection measurement in a statistically significant manner up to 1 month after injection. Later review did not show any further decrease in maximal mouth opening. Furthermore, there was no statistically significant difference in the mean lateral excursion at different postoperative periods. They performed injections twice in the joint without arthrocentesis and this may explain the conflict in the results with those that obtained in our study. In addition to, it proves the efficacy of arthrocentesis in management of internal derangement.

In the current study for assessment of clicking sound, the study groups were divided into patients with joints presented with clicking and others with joints not presented with clicking.The division of joints presented with clicking represent the joints of recent history of clicking within the last month before the procedure.Ingroup I, it was reported that 5 patients with joints presented without clicking (25\%) and 15 patients withjoints with clicking $(75 \%)$ of total 20 patients\&in the group II, it was reported that 2 patients with joints presented without clicking $(10 \%)$ and 18 patients withjoints with clicking $(90 \%)$ of total 20patients.After one month, in group I clicking was reported in $50 \%$ while in group II clicking where reduced in to $25 \%$.After three months, in group I clicking was reportedin $35 \%$ while in group II clicking where elevated to $35 \%$.After six months, in groupI clicking was reported in $25 \%$ while in group II clicking where continuous elevation to $50 \%$. Disappearance of the clicking postoperatively in all groups was suggested to be due to the effect of the arthrocentesis procedure as it dilutes inflammatory mediators and releases disc adhesion and lock inside the joint which facilitate disc gliding in the upper compartment which is proved by increased mouth opening and jaw movements to the normal range.

This explanation in accordance with Nitzan et al (1991),they stated lavage of the upper joint space reduces pain by removing inflammatory mediators from the joint, increasing mandibular mobility by removing intra-articular adhesions, eliminating the negative pressure within the joint, recovering disc and fossa space and improving disc mobility, which reduces the mechanical obstruction caused by the anterior position of disc.

Also it was in accordance with Yura et al (2011), As they stated that arthrocentesis under sufficient pressure can also remove adhesions, widen the joint spaces and improve mouth opening in patients who presented with limited mouth opening, significant improvement was seen in the immediate postoperative period and with reduction in pain, mouth opening further increased from third to sixth months and marginal improvement was observed with jaw deviation and clicking.

Mechanism of improvement in clicking sounds:

Although TMJ sounds are typically divided into two groups, clicks and crepitus, Prinz and Ng (1997) suggested that a classification into three basic groups could be more appropriate. Single short-duration sounds were termed clicks, multiple short-duration sounds were labelled creaks and all long-duration sounds were called crepitus.

The improvement in clicking sounds was attributed mainly to the injection of Sodium Hyaluronate following arthrocentesis. Marshall(1997) stated that sodium hyaluronate, a glycosaminoglycan produced and released by specialised synovial cells, is present in particularly high concentrations in the joint cartilage and synovial fluid. In normal conditions, this substance plays an important role in maintaining intra-articular homeostasis. It favours the elasticity and viscosity of the synovial fluid, providing a cushion against any shocks; it has a lubricating, anti-inflammatory and pain-relieving action and enables the tissue repair processes to be activated in the cartilage with a normalising action on the synthesis of endogenous acid by the synovial cells.

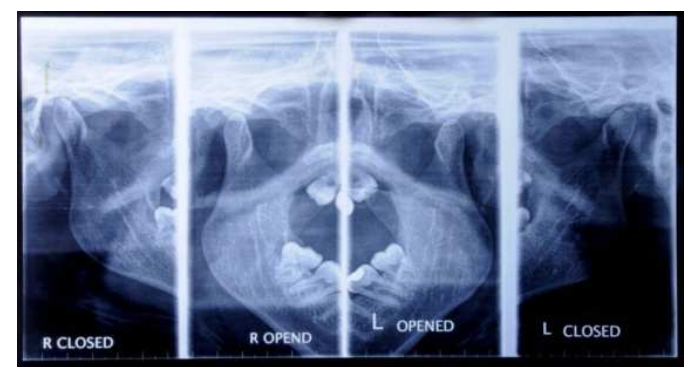

Figure 1. Digital X-ray of TMJ (close and openmouth): Right: TMJ with relatively limited movement of the joint(Bony density is seen at posterior part of jointspace) Left : TMJ shows normal radiographic appearance.

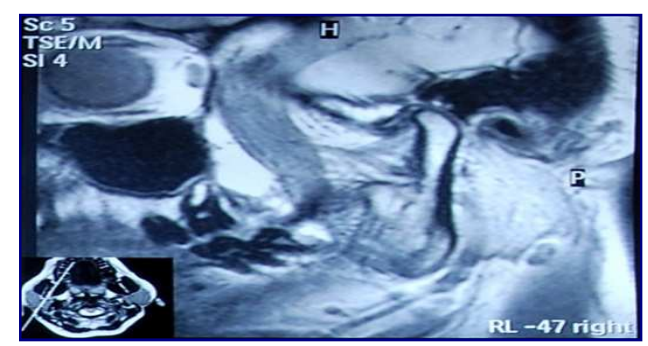

Figure 2. Preoperative MRI in closed mouth position, evidence of anterior disc displacement with reduction. 


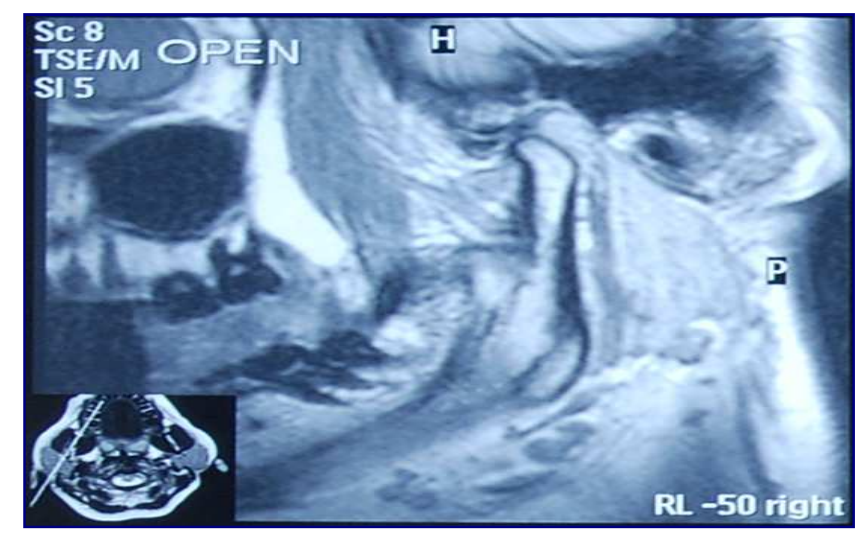

Figure 3. Preoperative MRI in open mouth position, evidence of anterior disc displacement with reduction.

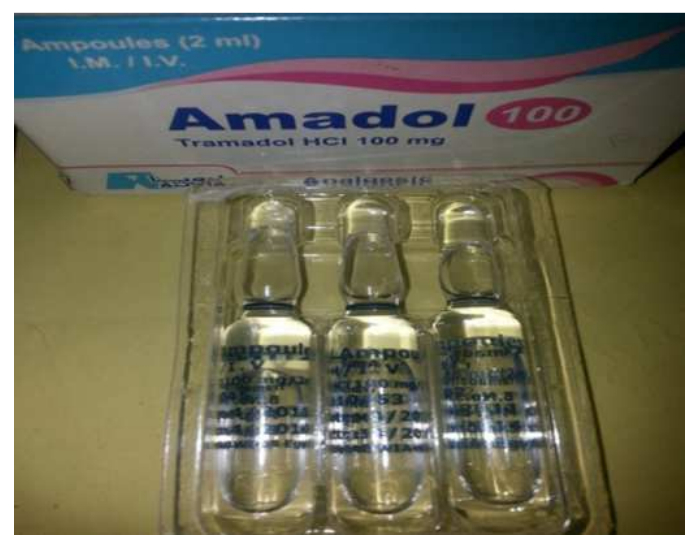

Figure 4. (Amadol ampoules I.M/I.V 100mg/2ml).produced by ADWAIAEYGPT.

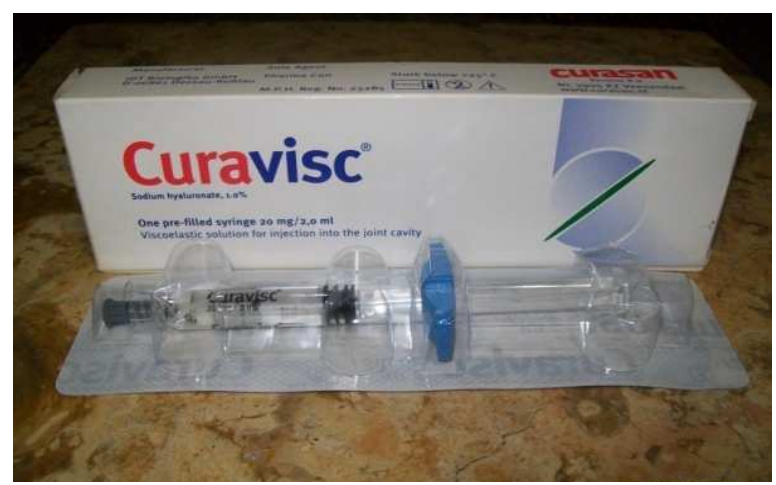

Figure 5. (curavisc 20mg / $2 m l$ syringe) by IDTBiologika GmbH campany, Germany.

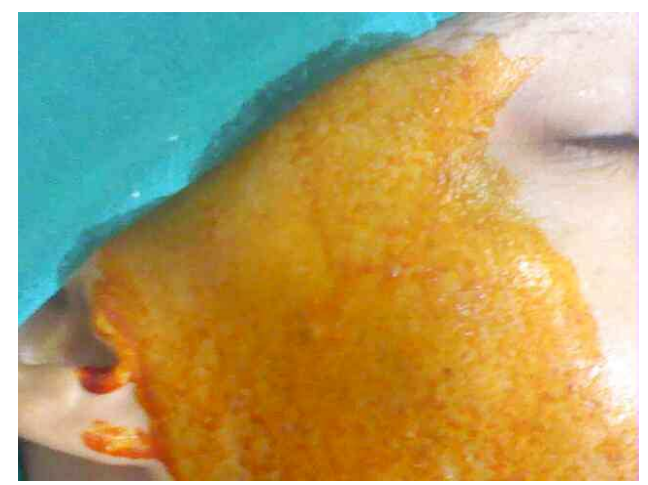

Figure 6. Preparation of the operative site for arthrocentesis.

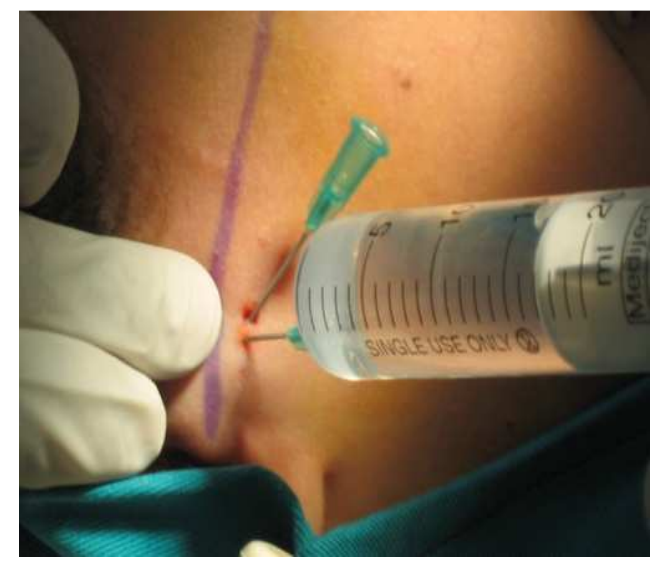

Figure 7. Injection of the saline solution into the upper joint compartment during the process ofarthrocentesis.

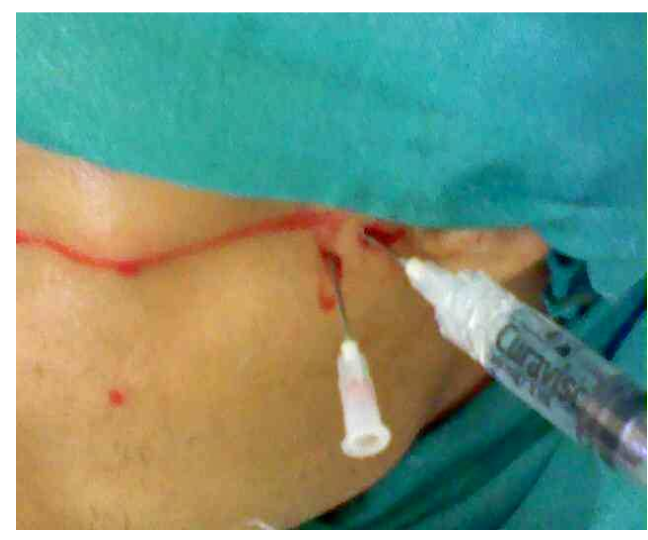

Figure 8. Injection of sodium hyaluronate in upper compartment of the joint whichcommercially available Sodium Hyaluronate(curavisc 20mg / $2 \mathrm{ml}$ syringe)by IDTBiologika GmbH campany, Germany.

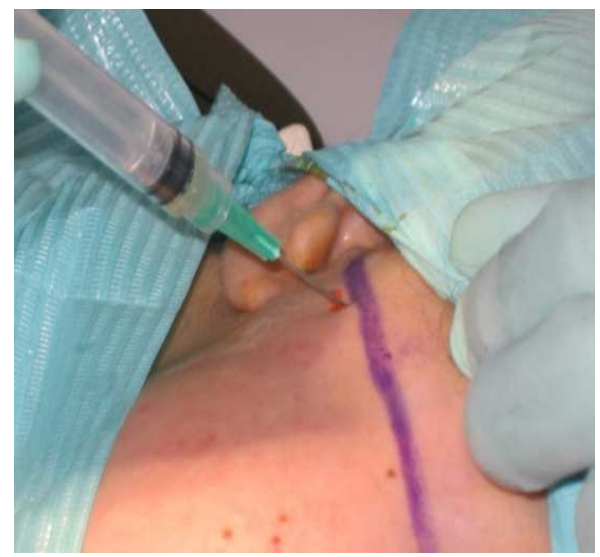

Figure 9. Injection of the tramadol into the upperjoint compartment following the process of arthrocentesis.

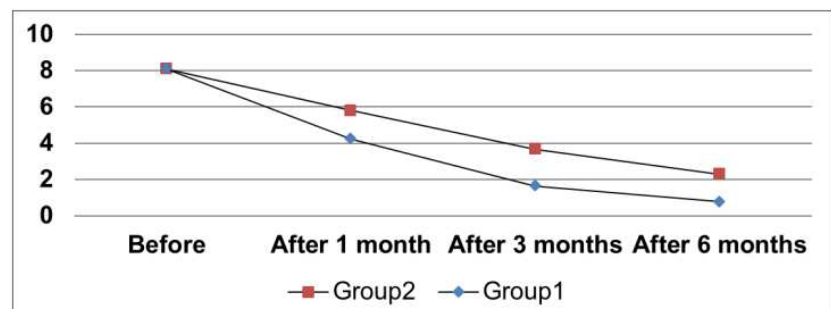

Figure 10. Pain levels for the two study groups and changes for each group along the study period. 


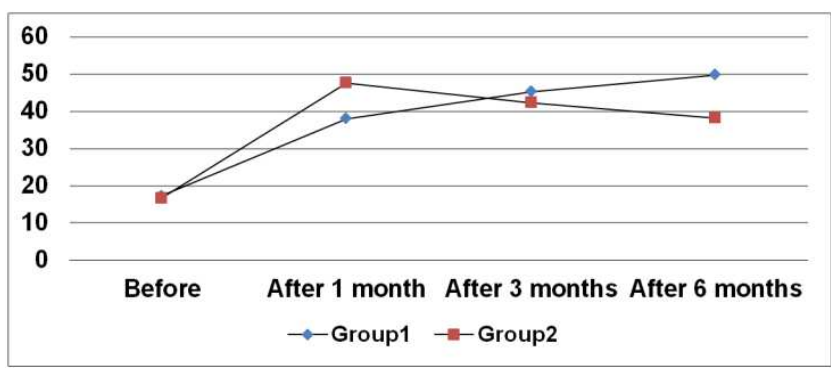

Figure 11. Changes in the maximal mouth opening for the study groups.

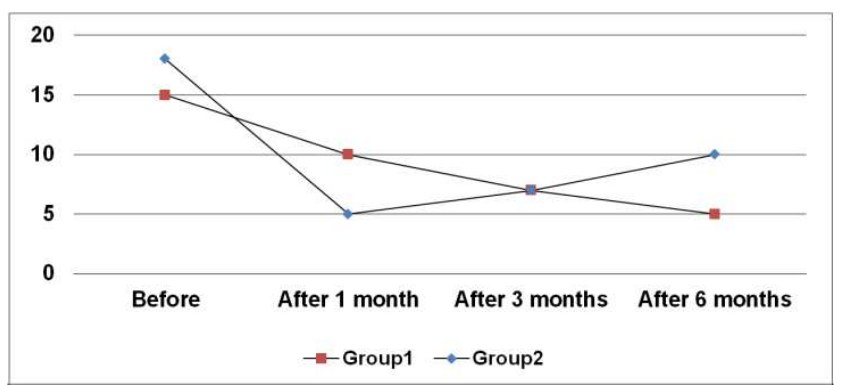

Figure 12. Graph showing the clicking sounds in the study groups for the joints that presented with clicking.

Table 1. Pain levels for the two study groups and changes for each group along the study period.

\begin{tabular}{|c|c|c|c|c|c|}
\hline \multicolumn{3}{|c|}{ Independent T-test } & \multirow{2}{*}{$\begin{array}{l}\text { Group II } \\
\text { Mean } \pm \text { SD }\end{array}$} & \multirow{2}{*}{$\begin{array}{l}\text { Group I } \\
\text { Mean } \pm \text { SD }\end{array}$} & \multirow{2}{*}{ Pain } \\
\hline Sig & P-Value & $\mathbf{T}$ & & & \\
\hline n.s. & 1.000 & 0.000 & $8.10 \pm 0.79$ & $8.10 \pm 0.91$ & Before \\
\hline h.s. & 0.000 & -6.941 & $5.80 \pm 0.77$ & $4.25 \pm 0.64$ & After 1 month \\
\hline h.s. & 0.000 & -12.924 & $3.65 \pm 0.49$ & $1.65 \pm 0.49$ & After 3 months \\
\hline h.s. & 0.000 & -7.451 & $2.30 \pm 0.66$ & $0.80 \pm 0.62$ & After 6 months \\
\hline
\end{tabular}

Table 2. Changes in the maximal mouth opening for the study groups.

\begin{tabular}{llllll}
\hline \multicolumn{2}{l}{ Independent T-test } & & Group II & Group I & Mouth opening \\
\hline Sig & P-Value & t & Mean \pm SD & Mean \pm SD & Before \\
\cline { 4 - 5 } n.s. & 0.542 & 0.616 & $16.70 \pm 3.29$ & $17.30 \pm 2.85$ & After 1 month \\
h.s. & 0.000 & -8.718 & $47.50 \pm 3.69$ & $37.95 \pm 3.22$ & After 3 months \\
h.s. & 0.007 & 2.873 & $42.35 \pm 3.65$ & $45.30 \pm 2.79$ & After 6 months \\
h.s. & 0.000 & 12.506 & $38.15 \pm 3.54$ & $49.80 \pm 2.19$ & \\
\hline
\end{tabular}

Table 3. Preoperativepresence (positive) and absence (negative) of clicking sounds in the study groups.

\begin{tabular}{lllll}
\cline { 1 - 3 } Groups & & & \multirow{2}{*}{ Clicking } & \\
\hline Total & Group II & Group I & & Positive \\
83 & 18 & 15 & $\mathrm{~N}$ & \\
7 & 90.00 & 75.00 & $\%$ & Negative \\
17.50 & 2 & 5 & $\mathrm{~N}$ & \\
40 & 10.00 & 25.00 & $\%$ & Total \\
100.00 & 20 & 20 & $\mathrm{~N}$ & \\
\hline
\end{tabular}

Table 4. Presence (positive) and absence (negative) of clicking sounds in the study groups after one month postopertive.

\begin{tabular}{lllll}
\cline { 1 - 3 } Groups & & & \multirow{2}{*}{ Clicking } & \\
\cline { 1 - 4 } Total & Group II & Group I & & \\
37.50 & 5 & 10 & $\mathrm{~N}$ & Positive \\
25 & 25.00 & 50.00 & $\%$ & \\
62.50 & 15 & 10 & $\mathrm{~N}$ & Negative \\
40 & 75.00 & 50.00 & $\%$ & \\
100.00 & 20 & 20 & $\mathrm{~N}$ & Total \\
\hline
\end{tabular}

Table 5. Presence (positive) and absence (negative) of clicking sounds in the study groups after three months postoperative.

\begin{tabular}{|c|c|c|c|c|}
\hline \multicolumn{3}{|l|}{ Groups } & \multirow{2}{*}{ Clicking } & \\
\hline Total & Group II & Group I & & \\
\hline 14 & 7 & 7 & $\mathrm{~N}$ & \multirow{2}{*}{ Positive } \\
\hline 35.00 & 35.00 & 35.00 & $\%$ & \\
\hline 26 & 13 & 13 & $\mathrm{~N}$ & \multirow{2}{*}{ Negative } \\
\hline 65.00 & 65.00 & 65.00 & $\%$ & \\
\hline 40 & 20 & 20 & $\mathrm{~N}$ & \multirow{2}{*}{ Total } \\
\hline 100.00 & 100.00 & 100.00 & $\%$ & \\
\hline
\end{tabular}

Table 6. Presence (positive) and absence (negative) of clicking sounds in the study groups after six month postoperative.

\begin{tabular}{|c|c|c|c|c|}
\hline \multicolumn{3}{|l|}{ Groups } & \multirow{2}{*}{ Clicking } & \\
\hline Total & Group II & Group I & & \multirow{3}{*}{ Positive } \\
\hline 15 & 10 & 5 & $\mathrm{~N}$ & \\
\hline 37.50 & 50.00 & 25.00 & $\%$ & \\
\hline 25 & 10 & 15 & $\mathrm{~N}$ & \multirow{2}{*}{ Negative } \\
\hline 62.50 & 50.00 & 75.00 & $\%$ & \\
\hline 40 & 20 & 20 & $\mathrm{~N}$ & \multirow{2}{*}{ Total } \\
\hline 100.00 & 100.00 & 100.00 & $\%$ & \\
\hline
\end{tabular}

\section{Conclusion}

It has been concluded that

1. Arthrocentesis is an effective conservative procedure in treatment of temporomandibular joint internal derangement.

2. Clinical efficacy of arthrocentesis with tramadolhydrochloride in the temporomandibular joint internal derangements.

3. Safty of tramadol intraarticular injection in the temporomandibular joint.

\section{References}

[1] Alkan A and Kilic E: A new approach to arthrocentesis of the temporomandibular joint, Int J Oral Maxillofac Surg 38: 85 $86,2008$. 
[2] Bjørnland T, Gjaerum AA, Møystad A: Osteoarthritis of the temporomandibular joint: an evaluation of the effects and complications of corticosteroid injection compared with injection with sodium hyaluronate.J Oral Rehabil.; 34(8):5839, 2007.

[3] El-Sharrawy EA, Hakim IE, Sameeh E. Attenuation of Creactive protein increases after exodontia by tramadol and ibuprofen. AnesthProg 2006;53:78-82.

[4] EmshoffR:: Clinical factors affecting the outcome of arthrocentesis and hydraulic distension of the temporomandibular joint. Oral Surg Oral Med Oral Pathol Oral RadiolEndod; 100:409-14.2005

[5] Emshoff R., Rudisch A.: Determining predictor variables for treatment outcomes of arthrocentesis and hydraulic distention of the temporomandibular joint, J Oral MaxillofacSurg 62(7):816-23, 2004.

[6] Escoda-Francolí J, Vázquez-Delgado E, Gay-EscodaC. : Scientificevidence on the usefulness of intraarticular hyaluronic acid injection in the management of temporomandibular dysfunction. Med Oral Patol Oral Cir Bucal. 1;15(4):644-8. 2010

[7] Guarda-Nardini L. Stifano M, BrombinC,SalmasoL,Manfredini D.:A one year case series of arthrothentesis with hyaluronic acid injection for tempromandiblar joint osteoarthritis, OralSurg Oral Med Oral PatholOral Radiol Endod,1039(6):14-22,2007.

[8] Gulen H, Ataoglu H, Haliloglu S, Isik K.: Proinflammatory cytokines in temporomandibular joint synovial fluid before and after arthrocentesis. Oral Surg Oral Med Oral Pathol Oral Radiol Endod.; 107(5): 1010-1016. 2009

[9] Hanife A Hilal A. Ahmet BIsil O TYetkin OAhmet E:The effect of intraarticular combinations of tramadol and ropivacaine with ketamine on postoperative pain after arthroscopic meniscectomy,ArchOrthop Trauma Surg (2010) 130:307-312

[10] Kaneyama K, Segami N, Nishimura M,SuzukiT,Sato J,Fujimura K.: Comparison of Cytokine Level in Synovial Fluid Between Successful and Unsuccessful Cases in Arthrocentesis of the Temporomandibular Joint,J Oral MaxillofacSurg 62:284-287, 2004.

[11] Kapral S, Gollmann G, Walt B et al: Tramadol added to mepivacaine prolongs the duration of axillary plexus blockage. AnesthAnalg1999,88:853-856

[12] Laskin D. M. : Arthrocentesis for the Treatment of Internal Derangements of the Temporomandibular Joint, Alpha Omegan 102 ( 2 ): 46 - 50, 2009.

[13] Manfredini D., Rancitelli D.,Ferronato G. \&Guarda-NardiniL.:
Arthrocentesis with or without additional drugs in temporomandibular joint inflammatory degenerative disease: comparison of six treatment protocols.J Oral Rehabil.; 39(4):245-51, (2012)

[14] Marshall K.W.: The current status of Hylan therapy for the treatment of osteoarthritis, Today's Therapeutic Trends 15: 99-108, 1997.

[15] Monje-Gil F, Nitzan D, González-Garcia R.: Temporomandibular joint arthrocentesis. Review of the literature. Med Oral Patol Oral Cir Bucal.; 1:17(4): 575-81, 2012.

[16] Neeli AS, Umarani M, Kotrashetti SM, Baliga S.: Arthrocentesis for the treatment of internal derangement of the temporomandibular joint. J Maxillofac Oral Surg.;9(4):350-4,2010

[17] Nitzan D.W., Dolwick M., Martinez G.: Temporomandibular joint arthrocentesis: A Simplified treatment for sever limited mouth opening, J Oral MaxillofacSurg 48: 1163, 1991.

[18] Prinz J.F., NG K.W.:Classification of TMJ Sounds, Archives of Oral Biology, 41, 631, 1997.

[19] Roy M. F, Jacques R. C, Sanford H. R, John R.P. T, William O, and Marc K: Tramadol for the Treatment of joint Pain Associated with Osteoarthritis: A Randomized,Double-Blind, Placebo-Controlled Trial, current therapeutic research'VOL. 62, No. 2. FEB 2001.

[20] Tamer A. H:Arthrocentesis with Injection of Tramadol and Cox-2 Inhibitor for the Management of Internal Derangements of the Tempromandibular Joint (A Comparative Study) Journal of American Science,66-72, $2012 ; 8(1)$

[21] Van Loon J P, Grauw J C, Brunott A, WeertsE A, Van Weber P $\mathrm{R}$ :Upregulation of articular synovial membrane 1-opioid-like receptors in an acute equine synovitis model,The Veterinary Journal.550-558, (2012).

[22] Yeung RW, Chow RL, Samman N, Chiu K.: Short-term therapeutic outcome of intra-articular high molecular weight hyaluronic acid injection for non-reducing disc displacement of the temporomandibular joint. Oral Surg Oral Med Oral Pathol Oral RadiolEndod.; 102(4):453-61,2006.

[23] Yura S, Ooi K, Izumiyama Y.: Relationship between the Effectiveness of Arthrocentesisunder Sufficient Pressure and Conditions of the Temporomandibular Joint. ISRN Dentistry; 2011, 5 pages. 2011.

[24] Yura S., Totsuka Y.: Relationship between effectiveness of arthrocentesis undersufficient pressure and conditions of the temporomandibular joint, J Oral MaxillofacSurg 63(2):225$228,2005$. 